\title{
Cryogenic Design and Performance of an Apparatus for Magnetically Trapping Ultracold Neutrons
}

\author{
P. R. Huffman ${ }^{\mathrm{a}, \mathrm{b}, *}$, K. J. Coakley ${ }^{\mathrm{c}}$, J. M. Doyle ${ }^{\mathrm{d}}$, C. R. Huffer ${ }^{\mathrm{a}, \mathrm{b}}$, H. P. Mumme, C. M. O’Shaughnessy, ${ }^{\mathrm{f}, \mathrm{b}}$, K. W. Schelhammer ${ }^{\mathrm{a}, \mathrm{b}}$, \\ P-N. Seo ${ }^{b}$, L. Yang ${ }^{g}$
}

\author{
${ }^{a}$ North Carolina State University, 2401 Stinson Drive, Box 8202, Raleigh, NC 27695 \\ ${ }^{b}$ Triangle Universities Nuclear Laboratory, 116 Science Drive, Box 90308, Durham, NC 27708 \\ ${ }^{c}$ National Institute of Standards and Technology, 325 Broadway, Boulder, CO 80305 \\ ${ }^{d}$ Harvard University, 17 Oxford St., Cambridge, MA 02138 \\ ${ }^{e}$ National Institute of Standards and Technology, 100 Bureau Drive, Stop 8461, Gaithersburg, MD 20899 \\ ${ }^{f}$ University of North Carolina at Chapel Hill, 120 E. Cameron Ave., CB \#3255, Chapel Hill, NC 27599 \\ ${ }^{g}$ University of Illinois at Urbana-Champaign, 1110 West Green Street, Urbana, IL 61801
}

\begin{abstract}
The cryogenic design and performance of an apparatus used to magnetically confine ultracold neutrons (UCN) is presented. The apparatus is part of an effort to measure the beta-decay lifetime of the free neutron and is comprised of a high-current superconducting magnetic trap that surrounds $\sim 211$ of isotopically pure ${ }^{4} \mathrm{He}$ cooled to approximately $250 \mathrm{mK}$. A $0.89 \mathrm{~nm}$ neutron beam can enter the apparatus from one end of the magnetic trap and a light collection system allows visible light generated within the helium by decays to be transported to detectors at room temperature. Two cryocoolers are incorporated to reduce liquid helium consumption.
\end{abstract}

Keywords: neutron lifetime, magnetic trap, cryocooler, ultracold neutrons

\section{Introduction}

The neutron lifetime, $\tau_{n}$, plays an important role in both nuclear astrophysics and in furthering the understanding of weak interactions in the Standard Model. It is an important experimental parameter in theoretical predictions of the primordial abundance of ${ }^{4} \mathrm{He}$ in Big Bang Nucleosynthesis[1,2]. A precision measurement of $\tau_{n}$ also provides a self-consistency check of the unitarity of the CKM mixing matrix, which relates the weak and mass eigenstates of quarks in the Standard Model[3]. Recent discrepancies in reported values of $\tau_{n}$ suggest unknown systematics are present in at least some of the existing analyses[4].

As part an effort to measure the neutron lifetime using a new experimental technique[5,6], a cryogenic apparatus capable of cooling $\sim 21$ liters of isotopically pure ${ }^{4} \mathrm{He}$ to approximately $250 \mathrm{mK}$ has been designed and constructed. The system must accommodate both a vertically-oriented dilution refrigerator to cool the helium within the measurement cell, and a $4.2 \mathrm{~K}$ liquid helium bath for cooling the approximately $1.5 \mathrm{~m}$ long horizontally-oriented superconducting magnetic trap. In addition, the cryostat is designed to accommodate a $0.89 \mathrm{~nm}$ neutron beam entering horizontally from one end of the magnetic trap and on the other end, a light collection system that transports visible light (from neutron decay) from the nominal $250 \mathrm{mK}$ helium to detectors at room temperature.

\footnotetext{
${ }^{*}$ Corresponding author

Email address: paul_huffman@ncsu.edu (P. R. Huffman)
}

This paper describes both the cryogenic design and performance of this apparatus.

\section{Cryostat Design}

The design of the apparatus incorporates a horizontal dewar with vertical towers at each end to accommodate a dilution refrigerator and a set of magnet current leads. A cross-sectional schematic of the apparatus is shown in Fig. 1.

The dewar was designed by our group and fabricated jointly with a commercial vendor. It consists of three concentric shells, each at successively lower temperatures. The outer, room temperature aluminum shell $(300 \mathrm{~K})$ provides the primary vacuum and safety enclosure for the apparatus. Inside reside the aluminum liquid nitrogen jackets and thermal shields $(77 \mathrm{~K})$, and inside that reside the stainless steel liquid helium jacket and the coldest, $4.2 \mathrm{~K}$, shields. Both cryogenic shields are covered with multiple layers of aluminized mylar insulation. The innermost section of the cryostat is the inner vacuum can (IVC) that contains the dilution refrigerator and measurement cell. A common vacuum space that surrounds the $77 \mathrm{~K}$ and $4.2 \mathrm{~K}$ shields is denoted as the outer vacuum chamber (OVC).

The apparatus design is largely determined by the horizontally-oriented superconducting magnetic trap. The magnet sits in a liquid helium bath and operates at a temperature of $4.2 \mathrm{~K}$. A thin-walled, $14 \mathrm{~cm}$ inner diameter stainless steel tube extends through the central bore of the magnet and is used to extend the IVC into this central region. This tube is connected with bellows for strain relief to two larger vacuum spaces at 


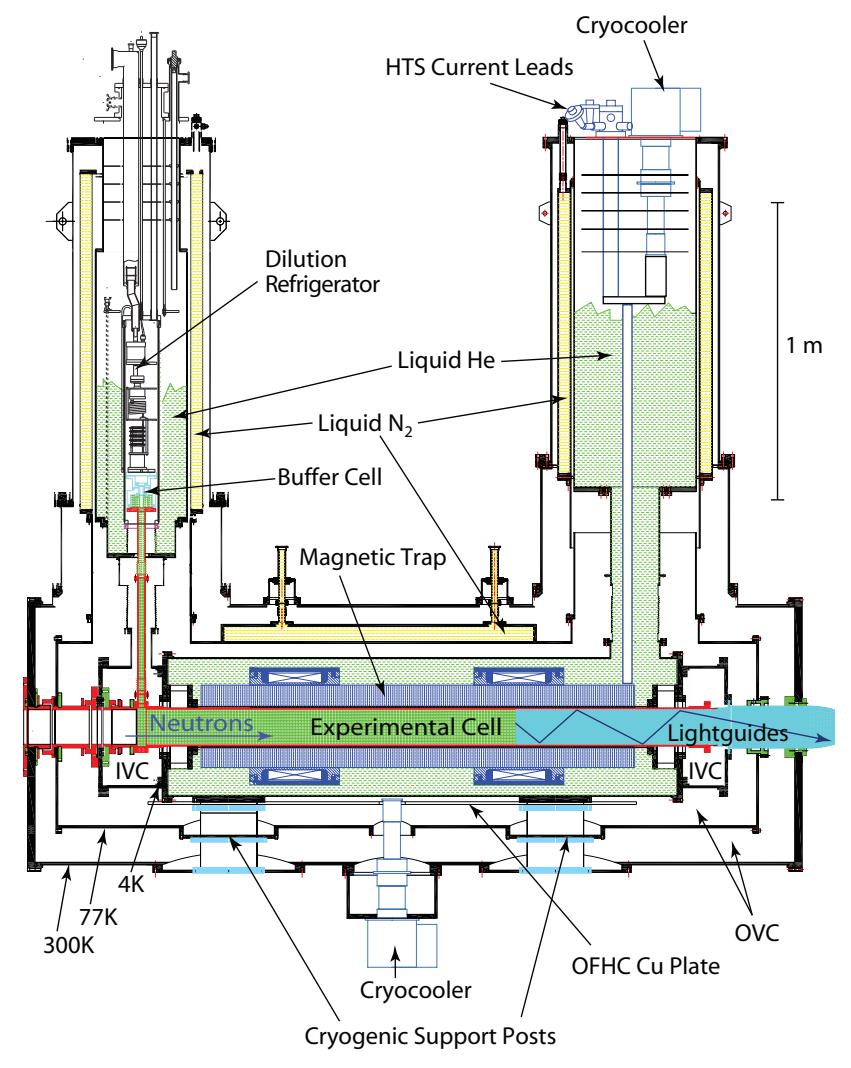

Figure 1: Cross-sectional schematic of the apparatus. The individual components are described in the text.

each end of the magnet that provide space for the cell support hardware and thermal connections. This same vacuum also extends to and surrounds the vertical ${ }^{3} \mathrm{He}-{ }^{4} \mathrm{He}$ dilution refrigerator.

The dilution refrigerator and associated gas handling system provides the primary cooling for the approximately 21 liters of liquid helium in the measurement cell. It is a Oxford Instruments model Kelvinox 400[7] with a measured cooling power of $400 \mu \mathrm{W}$ at $100 \mathrm{mK}$. The refrigerator is housed in the leftmost vertical tower shown in Fig. 1. This portion of the dewar is a standard $25.4 \mathrm{~cm}$ (10") diameter neck, nitrogen jacket cooled vertical dewar with a liquid helium volume that is separate from the helium surrounding the magnetic trap. This helium bath primarily provides cooling for the operation of the dilution refrigerator. Thermometers used at $4.2 \mathrm{~K}$ and below were either ruthenium oxide or thick-film chip resistors, both measured using commercial resistance bridges.

The right-most vertical tower shown in Fig. 1 is a standard $40.6 \mathrm{~cm}$ (16") diameter neck, nitrogen-cooled dewar that provides access for two sets of magnet current leads that extend to the magnet and serves as the helium reservoir for the magnet itself. Introducing 3,400 A into a liquid helium bath is done using high temperature superconducting (HTS) leads. This prototype set of leads was developed at Fermilab and is described in detail in reference [8]. Additional details on the HTS leads are given in section 3.1.1.

In the event that the magnet quenches, a large fraction of the liquid helium will boil as a portion of the magnet's energy is dissipated in the cryogenic bath. A $6.0 \mathrm{~cm}$ diameter springloaded cover plate is installed and used as a safety vent on the magnet lead tower to accommodate this exhaust. Although minimal boiloff is observed in the dilution refrigerator tower during a quench, a vendor-supplied safety vent is also present on the top flange.

Two Gifford-McMahon type Sumitomo RDK-415D cryocoolers, each with cooling powers of $1.5 \mathrm{~W}$ at $4.2 \mathrm{~K}$, are incorporated into the design to reduce helium consumption[7]. One is attached to the HTS leads in the vertical tower, and the second is attached below the horizontal dewar to remove heat from the two magnet support posts. Specifics of the two are discussed in sections 3.1.1 and 3.3.

\section{Superconducting Magnetic Trap}

The design, construction, and testing of the superconducting magnetic trap have been discussed in detail in a separate publication[9]. A photograph of the magnetic trap is shown in Fig. 2.

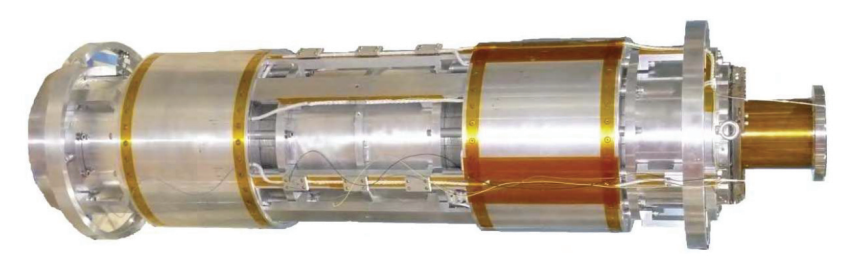

Figure 2: Photograph of the Ioffe-type superconducting magnetic trap. The length of the magnetic trap is $1.6 \mathrm{~m}$ and the diameter is $36 \mathrm{~cm}$. The smaller tube extending from the right-hand side is part of the IVC. Details of the construction and testing can be found in reference [9].

The large Ioffe-type magnetic trap consists of a quadrupole assembly that provides radial confinement and two solenoid assemblies with the same current sense that provide axial confinement. The high current quadrupole[10] (from the High Energy Accelerator Research Organization (KEK) institute in Japan) and the two low current solenoids are both wound using NbTi superconductor. The assembly was designed so that the quadrupole and solenoids would operate at $85 \%$ and $75 \%$ of the short-sample current in the superconducting wire, providing a trap depth of $3.1 \mathrm{~T}$. In initial testing of the assembly, these reached $74 \%$ and $70 \%$ respectively of their short-sample limits, thus corresponding to a trap depth of $2.8 \mathrm{~T}$ or $90 \%$ of the design depth. During our initial testing and characterization of the apparatus, the magnet was typically operated at $70 \%$ of the design depth, or $2.2 \mathrm{~T}$, in order to minimize the risk of quenches. Quench protection was incorporated into both the quadrupole and solenoid magnets.

As a result of its high operating current, heavy weight, and large volume, the KEK trap presented serious cryogenic challenges. In addition to the construction of the dewar itself shown in Fig. 1 to accommodate the magnet, it was necessary to use HTS leads to reduce the heat loads into the liquid helium to an acceptable level. Two pair of leads were used, one for the 
3,400 A needed for the quadrupole, and a smaller pair for the 250 A solenoids. Also, cryogenic posts were constructed to support the over $500 \mathrm{~kg}$ weight of the KEK trap, while adding minimal heat load to the liquid helium bath. These are described in the following sections.

\subsection{Current Leads}

\subsubsection{High-Current Leads}

The quadrupole and solenoids are powered separately and neither can be operated in persistent current mode due to the need to ramp the magnetic field to minimize systematic effects. Conventional vapor cooled current leads have a thermal performance limit of $1.2 \mathrm{~W} / \mathrm{kA}$ per lead[11]. To bring 3,400 A of current to the KEK quadrupole magnet with conventional leads would deposit at least $8.2 \mathrm{~W}$ of power into the liquid helium bath. With such a heat load, it would be too costly to operate the trap continuously.

We were able to employ a pair of 5,000 A HTS leads from Fermilab that reduced the heat load by a factor of six. The Fermilab HTS leads were developed to replace current leads at Fermilab's Tevatron[8]. The pair we obtained is a prototype pair developed by American Superconductor Corporation[7]. In addition, we also realized that using HTS leads for the $250 \mathrm{~A}$ solenoids could further reduce the liquid helium heat load. These leads are described in the next subsection.

The high-current HTS leads, as shown in Fig. 3, are constructed in three sections. The upper section is copper and provides a thermal gradient from room temperature to the HTS section. The middle section consists of parallel tapes of multifilamentary HTS superconductor in a silver alloy matrix. $\mathrm{Nb}_{3} \mathrm{Sn}$ low temperature superconducting (LTS) leads are then connected to the lower HTS section through a copper section that also acts as a thermal link to $4.2 \mathrm{~K}$.

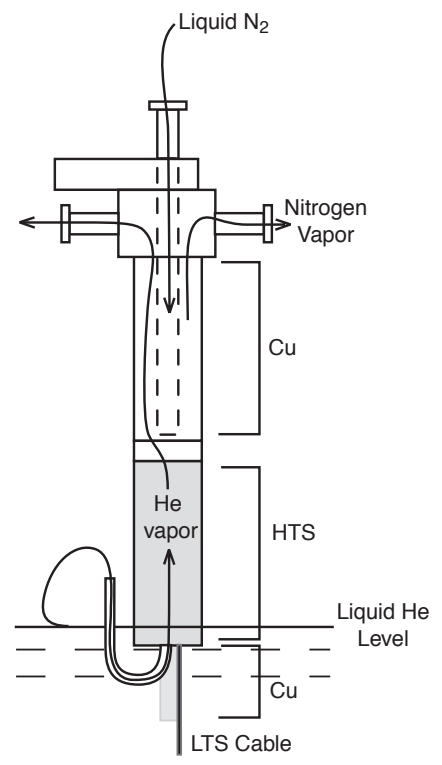

Figure 3: Cross-sectional view of the high temperature superconducting leads[8].
Since the HTS material must be kept below $80 \mathrm{~K}$ to remain superconducting, the copper-to-HTS junction is cooled with a continuous flow of liquid nitrogen. Cold nitrogen gas must thus flow at a rate of at least $0.7 \mathrm{~g} / \mathrm{s}(70 \mathrm{scfh})$ through the leads to maintain this temperature, consuming about 70 1/day. Our measured liquid nitrogen consumption of the system, including the transfer lines when operating at full magnet current, was somewhat higher, about 100 l/day.

To maintain the temperature below the critical point of the HTS material, one must also maintain a flow rate of $0.026 \mathrm{~g} / \mathrm{s}$ (19 scfh) of cold helium boil-off vapor through the current leads. This corresponds to a required minimum helium boiloff for the dewar of $16 \mathrm{l} /$ day.

The pair of HTS leads from Fermilab were tested in a $25.4 \mathrm{~cm}$ (10") diameter bore vertical dewar. The two leads, denoted as $\mathrm{A}$ and $\mathrm{B}$, were connected together at the bottom by a LTS cable. Four DC power supplies connected in parallel provided a maximum testing current of $3,410 \mathrm{~A}^{1}$. The temperatures at the $\mathrm{Cu}-\mathrm{HTS}$ and HTS-LTS junctions were monitored using resistance thermometers. Flow meters were installed at the nitrogen and helium gas exhausts to measure cryogen consumption. The leads were successfully tested up to 3,410 A.

Operational parameters of the current leads at 3,410 A are shown in Table 1. The voltage drop across the HTS section of Lead A was $1.5 \mathrm{mV}$, higher than the manufacturer's specification. This was a result of a repair to a broken solder joint where the manufacturer could not reattach the voltage tap directly to the HTS material itself. Instead the tap was soldered to the copper piece just above the HTS section. The measured voltage therefore included the additional voltage drop across a small section of copper. The measured liquid helium boiloff rate was 50 1/day, in agreement with data from a previous Fermilab test[8]. Note that during operation, one of the helium cooling lines was partially plugged, however we do not think this had a significant effect on the boiloff.

Table 1: Operational parameters of the Fermilab HTS Leads at 3,410 A.
\begin{tabular}{|l|c|}
\hline Liquid helium consumption, liter/day & 50 \\
Equivalent power to $4.2 \mathrm{~K}, \mathrm{~W}$ & 1.5 \\
Liquid nitrogen consumption, liter/day & 100 \\
HTS section voltage Lead A, mV & 1.8 \\
HTS section voltage Lead B, mV & 0.3 \\
Cu section voltage A \& B, mV & $\approx 20$ \\
\hline
\end{tabular}

To minimize heat loads and reduce the dependency on vapor cooling, a cryocooler was installed and thermally linked to the bottom copper section of the current leads to provide $1.5 \mathrm{~W}$ of cooling power at $4.2 \mathrm{~K}$. A thin layer of Kapton[7] is used to provide the necessary electrical isolation. The cryocooler in principle could reduce the helium consumption from the leads by as much as $50 \mathrm{l} /$ day. In addition, the cooling power from the first stage of the cryocooler was used to cool the Cu-HTS junctions of the pair of low-current HTS leads for the solenoids discussed below.

\footnotetext{
${ }^{1}$ Model number HP6680A[7]. The solenoids were powered by two HP6681A[7] supplies in parallel.
} 


\subsubsection{Low-current leads}

Our calculations showed that by using HTS instead of vaporcooled current leads for the $250 \mathrm{~A}$ solenoids, one could reduce the heat load to $4.2 \mathrm{~K}$ from $1.2 \mathrm{~W}$ to $0.2 \mathrm{~W}$. Such low-current HTS leads can be implemented by connecting commercially available HTS tape leads to custom designed copper rods.

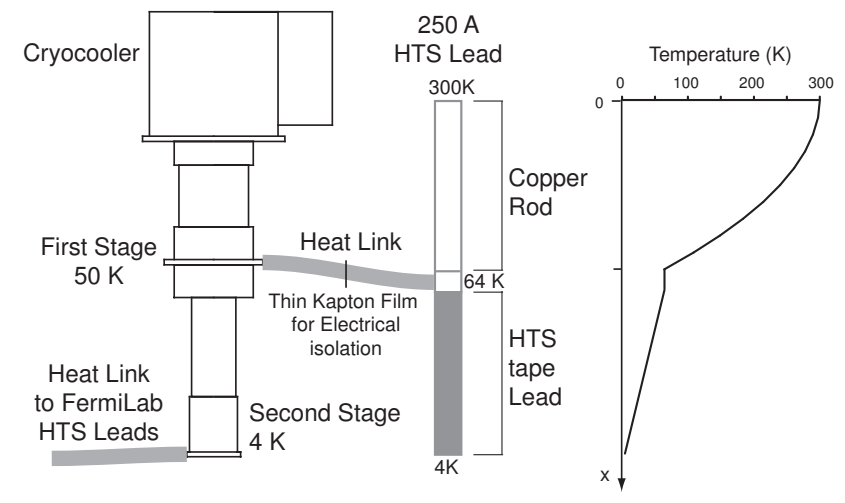

Figure 4: Schematic of the 250 A HTS lead. The Cu-HTS junction of the lead is cooled by the first stage of the $1.5 \mathrm{~W}$ cryocooler. The graph on the right shows the calculated temperature distribution across the lead.

A schematic of a 250 A HTS lead is shown in Figure 4. A $13.7 \mathrm{~cm}$ long copper wire brings the current from room temperature to the $\mathrm{Cu}-\mathrm{HTS}$ junction. Then a $30 \mathrm{~cm}$ long multifilamentary HTS tape with operating current of 250 A conducts the current to $4.2 \mathrm{~K}$. The $\mathrm{Cu}$-HTS junction has to be cooled to below $64 \mathrm{~K}$ for the proper operation of the lead. The heat load onto the junction comes from both the ohmic and conduction heating of the copper rod. Neglecting the effect of vapor cooling, we estimate the thermal balance of the copper rod using

$$
\frac{d\left(k A \frac{d T}{d x}\right)}{d x}+\frac{\rho I^{2}}{A}=0
$$

where $k$ is the thermal conductivity of copper, $A$ is the crosssection of the copper rod, $T$ is temperature, $\rho$ is the resistivity of copper and $I$ is current.

To simplify, we assume the copper rod has a uniform crosssection and $k$ and $\rho$ are independent of temperature. Then Eq. 1 has a simple solution

$$
T(x)=T(0)-\frac{\rho I^{2} x^{2}}{2 k A^{2}}+\left(\frac{\rho I^{2} L}{2 k A^{2}}-\frac{T(0)-T(L)}{L}\right) x,
$$

where $L$ is the total length of the copper rod. The heat load onto the Cu-HTS junction $Q$ can be calculated as

$$
Q=-\left.k A \frac{d T}{d x}\right|_{L}=\frac{\rho I^{2} L}{2 A}+\frac{(T(0)-T(L)) k A}{L} .
$$

$Q$ has a minimum value of $\sqrt{\rho k(T(0)-T(L)) / 2} I$ at $A / L=$ $\sqrt{\rho /(2 k(T(0)-T(L))} I$. Copper is chosen as the lead material precisely because it minimizes the product of resistivity and thermal conductivity, $\rho k$. Using the boundary conditions $T(0)=300 \mathrm{~K}$ and $T(L)=64 \mathrm{~K}$, the average thermal conductivity $k=6 \mathrm{~W} / \mathrm{cm} \mathrm{K}$ and the average resistivity $\rho=9 \times 10^{-7} \Omega \mathrm{cm}$, we obtain that $Q=6.3 \mathrm{~W}$, and $A / L=4.5 \times 10^{-3} \mathrm{~cm}$ for a $250 \mathrm{~A}$ lead. The results from this simplified model is very close to a numerical solution of Eq. 1 taking into full consideration of the temperature dependence of $k$ and $\rho$.

The $\mathrm{Cu}$-HTS junctions are cooled by the first stage of the $1.5 \mathrm{~W}$ cryocooler used to cool the HTS-LTS junctions of the Fermilab leads. The first stage has $45 \mathrm{~W}$ of cooling power at $50 \mathrm{~K}$, more than enough for the $12.6 \mathrm{~W}$ heat load at the two 250 A lead junctions. Electrical isolation is achieved with 5 mil thick Kapton[7] film.

At the copper to HTS junction, measured temperatures ranged from $53 \mathrm{~K}$ to $58 \mathrm{~K}$ with no current applied. During 200 A operation, the temperature rose to between $55 \mathrm{~K}$ and $60 \mathrm{~K}$. At the HTS-LTS junction, the temperatures were $7.0 \mathrm{~K}$ and $7.3 \mathrm{~K}$ at $0 \mathrm{~A}$ and $200 \mathrm{~A}$ respectively.

\subsection{Quench Protection}

At operating current, the energy stored in the quadrupole magnet is $336 \mathrm{~kJ}$ and in each solenoid is $180 \mathrm{~kJ}$. Such large stored energies, if deposited in a small section of the magnet during a quench, can cause permanent damage to the winding, possibly even vaporizing a small section of wire. Quench protection circuitry was designed to quickly dump the stored energy into an external resistor or the helium bath in order to protect the quadrupole and solenoid magnets in the event of a quench.

The quench protection circuit built for the low-inductance quadrupole magnet $(58.0 \mathrm{mH}$ [12]) followed a design by Fermilab[13], in which silicon controlled rectifiers (SCR) are used for fast current switching. The connection between the anode and cathode normally does not conduct current, but when a quench is detected, the dump SCR $\left(\mathrm{S}_{\mathrm{D}}\right)$ can be switched into a conductive state by a positive bias voltage applied to the gatecathode junction. A bank of four $100 \mu \mathrm{F}$ capacitors connected in parallel then discharges through $\mathrm{S}_{\mathrm{D}}$. The capacitors are initially kept at $350 \mathrm{~V}$ by an external high voltage power supply. The discharge current temporarily stops the current flow in the run SCRs for roughly $40 \mu \mathrm{s}$, long enough to turn all run SCRs into a non-conductive state. After the capacitors are fully discharged, current can only flow through an external dump resistor, where the majority of the stored energy of the magnet is dissipated. The dump resistor is constructed from $1.6 \mathrm{~cm}$ thick 316 stainless steel plate. It has a resistance of $100 \mathrm{~m} \Omega$, resulting in a characteristic energy dump time of $L / R=0.6 \mathrm{~s}$. The mass of the resistor, about $14 \mathrm{~kg}$, is large enough to limit its temperature rise after a quench to less then $50{ }^{\circ} \mathrm{C}$.

This technique however is only effective for low inductance magnets and will not work for the solenoids with inductances of $7 \mathrm{H}$ each. Instead, the solenoids are protected passively by diodes across six subdivisions of the magnet. During a quench, when the voltage across a subdivision reaches $2 \mathrm{~V}$, the corresponding diode switches to a conducting mode, letting some current bypass the subdivision, therefore reducing its current density and heating. This passive technique has the disadvantage that it dumps all of the stored energy into the liquid helium resulting in rapid boiling of the liquid helium. 
During testing of the individual components, no natural quenches occurred in either the quadrupole or solenoid assemblies. Heaters installed on the quadrupole were used to induce a quench in order to test the quench protection circuitry and measure the efficiency of removing the stored energy. Over the current range of $500 \mathrm{~A}$ to $2,800 \mathrm{~A}$, the protection circuit consistently removes an average of $95 \%$ of the stored energy. A quench of the quadrupole and solenoids operating at $80 \%$ of the trap depth results in a rapid boiloff of between 1001 and 1201 of helium, followed by an increased boiloff rate for an additional ten to fifteen minutes. The majority of this heat is from the energy stored in the solenoids.

\subsection{Magnet Support and Cooling}

To support the $500 \mathrm{~kg}$ magnetic trap while minimizing the heat loads into the liquid helium bath, we implemented G-10 fiberglass-based cryogenic posts. Our cryogenic post design closely follows the design given in reference [14]. Schematic views of the post are shown in Fig. 5.
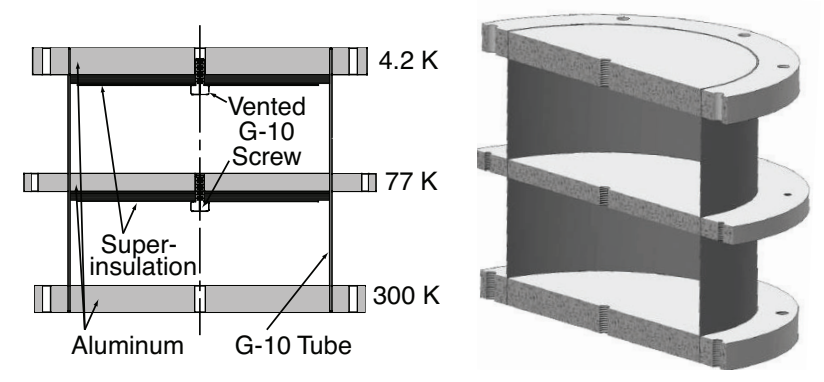

Figure 5: Two dimensional and three dimensional cross-section views of the $\mathrm{G}-10$ based cryogenic post. The posts are $19 \mathrm{~cm}$ tall.

The main body of the post is a $19 \mathrm{~cm}$ long, $19 \mathrm{~cm}$ diameter G-10 tube with a $1 \mathrm{~mm}$ wall thickness. Equally spaced aluminum flanges positioned at the bottom $(300 \mathrm{~K})$, middle $(77 \mathrm{~K})$ and top $(4.2 \mathrm{~K})$ of the tube provide lateral mechanical support for the tube as well as holes for attachments to the dewar. All flanges were shrunk-fit onto the tube. The inner diameter (ID) of each outer aluminum ring was machined to the exact outer diameter (OD) of the G-10 tube, while the OD of each inner metal disk was machined to be $0.5 \mathrm{~mm}$ more than the tube ID. During assembly, the outer rings were first positioned in place, then the inner disks were cooled to liquid nitrogen temperature (77 K) and slid into the tube. The inner $(77 \mathrm{~K})$ flange was first assembled using a spacer between the components and table to insure correct positioning. Small lips on the $300 \mathrm{~K}$ and $4.2 \mathrm{~K}$ inner flanges helped register them during assembly. Side surfaces of the rings and disks were roughened slightly using sand paper to increase the friction coefficient. As shown in Fig. 5, the $77 \mathrm{~K}$ and $4.2 \mathrm{~K}$ disks were covered with super-insulation to reduce blackbody radiation. The internal volumes were evacuated through vented G-10 screws in the center of each disk; tests showed that any vent holes on the side of the G-10 tube significantly reduced the load carrying capacity of the post.
A prototype post was built based the above design. It was load tested to $1,360 \mathrm{~kg}$ at room temperature using an active load. The heat load through the post was calculated from available G-10 thermal conductivity data and was estimated to be $(0.35 \pm 0.05) \mathrm{W}$ per post.

The magnet is supported from two posts as seen in Fig. 1. Due to thermal contraction, one post is allowed to move horizontally to minimize lateral stress. A thin sheet of Teflon[7] is used at the $300 \mathrm{~K}$ connection of the sliding post to reduce friction.

A large Oxygen-Free High Thermal Conductivity (OFHC)grade copper plate $25.4 \mathrm{~cm}$ (10.0") wide and $9.53 \mathrm{~mm}(3 / 8$ ") thick extends between the $4.2 \mathrm{~K}$ flanges of the posts and the lower half of the dewar in order to allow heat from conduction through the posts to be extracted externally without depositing it into the liquid helium reservoir. A Gifford McMahon-type cryocooler that provides $1.5 \mathrm{~W}$ of cooling power at $4.2 \mathrm{~K}$ is thermally linked to middle region of the copper bar. OxygenFree Electronic (OFE)-grade copper braiding is used to thermally link the cryocooler to the copper bar while providing vibration isolation in addition to allowing freedom for thermal contraction as the apparatus cools. Copper braids also connect the ends of the copper plate to the neutron entrance and light collection windows to remove conduction heat from these as well.

\section{Helium Filling}

The isotopically pure helium-4 used to fill the cell is stored at room temperature as a gas in an all-metal gas handling system. This helium passes through a liquid-helium cold trap before entering the cryostat in order to remove impurities. This section details the cryogenic design of the helium condensing lines, the expansion volume known as the "buffer cell", and the liquid-helium thermal link between the mixing chamber of the dilution refrigerator and the measurement cell.

\subsection{Helium Fill Lines}

The dilution refrigerator is equipped with two commerciallyinstalled lines that extend from the room temperature gas handling system to the mixing chamber area of the dilution refrigerator. These lines are $1.6 \mathrm{~mm}$ outer diameter stainless steel tubes that wrap around the still pumping line inside of the main helium bath, penetrate the $4.2 \mathrm{~K}$ IVC top, pass through the $1 \mathrm{~K}$ pot, are soldered to the outside of the still, and pass through the continuous heat exchangers before connecting to a volume attached to the mixing chamber denoted as the buffer cell. These fill lines have high impedance and are only used for filling the cell with helium. The measurement cell however must be pumped out prior to cooling down, which is done through a secondary line with smaller impedance.

A large heat load on the dilution refrigerator can result from superfluid film flow up this larger pumping line. These heat loads were minimized by heat-sinking this line to both the $50 \mathrm{mK}$ cold plate and the still of the dilution refrigerator. As shown in Fig. 6, the fill line has a pinhole aperture between 
these heat sinks to minimize film flow. This aperture is a $0.63 \mathrm{~mm}$ hole in a $0.13 \mathrm{~mm}$ thick copper foil soldered to cover the hole in a standard 1/8" stainless steel VCR gasket[7]. This hole has minimal impact on the impedance of the bypass line in the laminar flow regime. The gasket serves as a collection point for the small amount of refluxing helium, thus allowing the heat load to be taken up at the cold plate where the cooling power is higher rather than at the mixing chamber. When the system was cooled with the secondary fill line, the mixing chamber reached a base temperature of $50 \mathrm{mK}$ and $60 \mathrm{mK}$ without and with helium in the buffer cell, respectively.

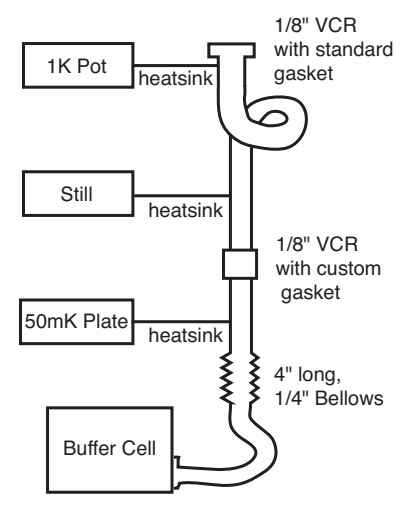

Figure 6: A schematic of the secondary pumping line.

\subsection{Buffer Cell}

The buffer cell is attached to the bottom of the mixing chamber and provides the thermal link between the the mixing chamber of the dilution refrigerator and superfluid helium heat link to the cell. It also serves as a reservoir to account for the thermal contraction of the liquid helium as it cools. This $10 \mathrm{~cm}$ diameter, $11 \mathrm{~cm}$ tall chamber (see Fig. 7) thermally connects the mixing chamber to the liquid helium through two copper fins coated with silver sinter. These provide a large surface area contact in order to minimize the thermal resistance between the mixing chamber and superfluid helium.

The buffer cell also contains a helium level sensor. This sensor is a capacitor formed from two concentric stainless steel tubes of length $11.4 \mathrm{~cm}$. The outer and inner tubes are $17.5 \mathrm{~mm} \mathrm{OD} \times 0.8 \mathrm{~mm}$ wall thickness and $19.1 \mathrm{~mm} \mathrm{OD} \times$ $1.7 \mathrm{~mm}$ wall thickness respectively and are separated by two Teflon[7] rings with vent holes at each end. The capacitance is $28 \mathrm{pF}$ and as the space between the two tubes fills with liquid helium, the capacitance changes by $2.3 \mathrm{pF}$. Condensation of the isotopically pure helium into the cell is controlled using this level meter so that the level of helium within the buffer cell is maintained approximately halfway up the fins during normal operation. Note that until the level of helium reaches the buffer cell, the measurement cell would not cool below $\sim 800 \mathrm{mK}$.

\subsection{Thermal link to the cell}

The thermal link between the buffer cell and measurement cell is provided by a continuous $2.54 \mathrm{~cm}$ diameter helium-filled

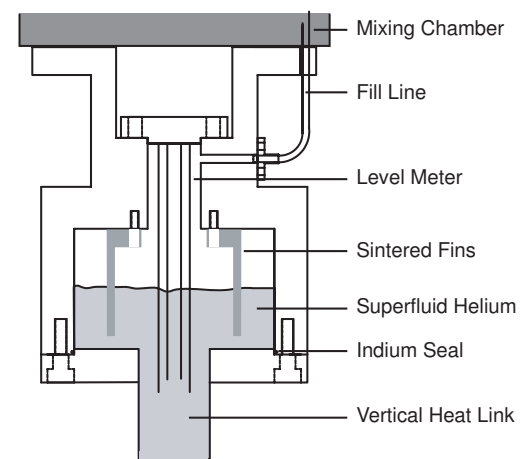

Figure 7: A cross-sectional view of the buffer cell. Copper fins coated with silver sinter maintain thermal contact between the mixing chamber of the dilution refrigerator and superfluid helium.

cupronickel $(\mathrm{CuNi})$ tube as can be seen in Figs. 1 and 8 . This design maximizes the thermal connection to mixing chamber while minimizing the amount of metal and thus effects from eddy-current heating when ramping the magnetic field. Forces arising from the thermal contraction of the CuNi tubing is minimized using two beryllium copper bellows.

Below $400 \mathrm{mK}$, the thermal conductivity through the helium is given by $[15,16]$

$$
\kappa=20 d T^{3}\left[\frac{\mathrm{W}}{\mathrm{K} \cdot \mathrm{cm}}\right]
$$

where $d$ is the diameter $(\mathrm{cm})$ of the ${ }^{4} \mathrm{He}$ column. The total length between the mixing chamber and the measurement cell is $88 \mathrm{~cm}$ that provides a calculated thermal conductivity of $0.8 \mathrm{~W} / \mathrm{K} \cdot \mathrm{cm}$ at $250 \mathrm{mK}$.

\section{Measurement cell}

The experimental cell is a cylindrical tube that contains the isotopically pure helium, scintillator, shielding, and first stage of the light transport system. The cell is positioned inside of the superconducting magnet assembly as shown in Fig. 1. Inside the cell, boron nitride $(\mathrm{BN})$ tubes are used as a neutron shield to prevent neutrons from absorbing on the stainless steel outer tube housing the cell. A thin tube of graphite is inside the $\mathrm{BN}$ to block light due to neutron-induced luminescence in the $\mathrm{BN}[17,18]$. Inside the graphite is a tetraphenyl butadiene (TPB) coated GoreTex[7] tube that is used to convert the $80 \mathrm{~nm}$ light produced from scintillations in the liquid helium to $430 \mathrm{~nm}$ light that can be extracted. Light is extracted from the cell using ultraviolet transmitting (UVT)-grade acrylic lightguides. The lightguide at the end of the cell also serves as the neutron beam stop.

The experimental cell is housed in a $1.8 \mathrm{~m}$ long, $12.7 \mathrm{~cm}$ diameter, $3.2 \mathrm{~mm}$ thick $316 \mathrm{~L}$ grade seamless stainless steel tube. The tube is positioned along the axis of the $1.6 \mathrm{~m}$ long quadrupole magnet with a radial clearance of $5 \mathrm{~mm}$. Due to the large size of the end flanges, it was not possible to pass an assembled geometry through the bore of magnet. The lightguide window flange was first soldered using $50 / 50 \mathrm{~Pb} / \mathrm{Sn}$ solder with 
a zinc-chloride based flux onto the tube in a vertical orientation while on the workbench. The cell was next inserted into position inside the magnet bore and the neutron entrance window flange was attached by soldering the flange assembly in place horizontally using the same solder and flux.

\subsection{Cell support}

It is critical that the supports for the cell suspend the $26 \mathrm{~kg}$ mass of the cell with little movement due to stretching of the mounts, while conducting minimal heat to the cell. Zylon[7] strands were used to support the cell from the $4.2 \mathrm{~K}$ shield. Zylon is a high strength, low creep, liquid crystalline material that can be spun into long fibers and has a room temperature ultimate tensile strength ${ }^{2}$ of $37 \mathrm{cN} / \mathrm{dtex}$.

Measurements at low temperature have shown that the thermal conductivity of Zylon fiber bundles spanning the temperature range $0.3 \mathrm{~K}$ to $4.2 \mathrm{~K}$ can be estimated by the properties of the fibers at $T=(0.7 \pm 0.1) \mathrm{K}[20]$. Our cell is suspended on each end of the IVC by three Zylon fiber bundles attached to the $4.2 \mathrm{~K}$ can. Each bundle has a linear mass density of 1656 dtex and are $8.25 \mathrm{~cm}$ long. They are affixed to the cell and to adjustable mounts attached to the $4.2 \mathrm{~K}$ can. The cell was then centered in the magnet bore by adjusting the positioning arms. The longitudinal position was fixed by a Vespel-22[7] spacer attached to the $4.2 \mathrm{~K}$ flange on the lightguide end. Based on measurements performed in reference [20], an individual $\mathrm{Zy}$ lon fiber bundle used in our experiment is estimated to impart a total of $0.17 \mu \mathrm{W}$ into the cell. The 6 total bundles thus impart a heat load of $1.0 \mu \mathrm{W}$.

\subsection{Neutron Entrance Windows}

The $0.89 \mathrm{~nm}$ neutron beam must travel from the exit of the neutron guide into the helium-filled measurement cell with minimal attenuation. In addition, the materials must not contain elements that will become activated by the neutron beam and must be opaque to blackbody radiation from room temperature surfaces to limit heating of the cell. A schematic of the neutron beam entrance design is shown in Fig. 8.

There are three vacuum windows that are each made from $20 \mathrm{mil}(508 \mu \mathrm{m})$ thick Teflon perfluoroalkoxy flouropolymer (PFA) film[21, 7]. Teflon-PFA is manufactured without metallic contaminants that could activate and produce background activation. Since PFA is a soft material that remains pliable even at cryogenic temperatures, it can be used as a gasket material to create self sealing windows on the room temperature $300 \mathrm{~K}$ vacuum flange, the $4.2 \mathrm{~K}$ flange separating the OVC and IVC, and the end of the experimental cell containing the superfluid helium. These windows efficiently pass neutrons, have minimal activation, and provide reliable vacuum seals. We have also successfully used Teflon Fluorinated Ethylene Propylene (FEP)[7] in the past with similar performance.

PFA provides minimal shielding of block blackbody radiation, so two additional windows made from $0.05 \mathrm{~mm}(0.002$ ”)

\footnotetext{
${ }^{2} \mathrm{~A}$ linear mass density of 1 tex is equivalent to a yarn mass of $1 \mathrm{~g} / \mathrm{km}$. High modulus Zylon has a density of $1.56 \mathrm{~g} / \mathrm{cm}^{3}$.
}

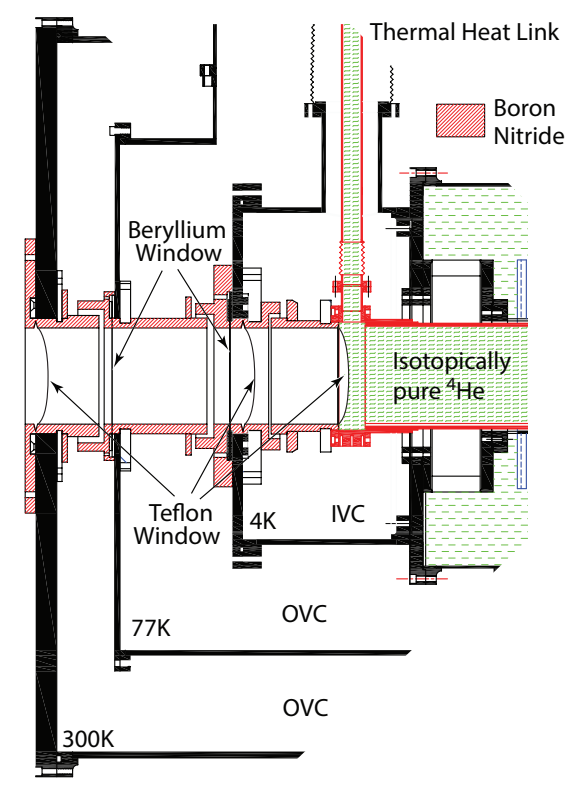

Figure 8: Side view of the neutron entrance and shielding materials.

thick, $16.5 \mathrm{~cm}(6.5$ ") diameter beryllium foil are included in the beam entrance. The $77 \mathrm{~K}$ foil prevents blackbody radiation from $300 \mathrm{~K}$ from reaching $4.2 \mathrm{~K}$, and a second foil at $4.2 \mathrm{~K}$ blocks the radiation originating at $77 \mathrm{~K}$ from reaching the experimental cell. The foils are thermally anchored at the edges to the to the cryostat end flanges with aluminum compression rings.

\subsection{Light Collection System}

The visible light signal generated in the measurement cell is transported out of the apparatus and detected using photomultiplier tubes at room temperature. This system was optimized for both maximum light output and minimum heat leakage into the measurement cell. We focus here on the cryogenic aspects of the system. A schematic of the light collection system is shown in Fig. 9.

Light from the measurement cell travels through an acrylic lightguide, exits the $250 \mathrm{mK}$ region through an acrylic window denoted as the "cell snout", passes through acrylic and single crystal quartz windows at $4.2 \mathrm{~K}$, and then is transported to the detectors through a lightguide spanning the region between the $77 \mathrm{~K}$ and $300 \mathrm{~K}$ shells.

The lightguide in the cell is an $11.6 \mathrm{~cm}$ dimeter, $70.3 \mathrm{~cm}$ long UVT-grade cast acrylic rod. It is positioned against the $250 \mathrm{mK}$ window at the end of the cell, but not attached. This rod is immersed in the liquid helium contained within the measurement cell and is cooled through the helium thermal link discussed above.

A window at the end of the cell is required to both contain the isotopically pure helium within the cell as well as allow the light to be transmitted to room temperature. We are using the cylindrical "snout" geometry shown in Fig. 10 containing 


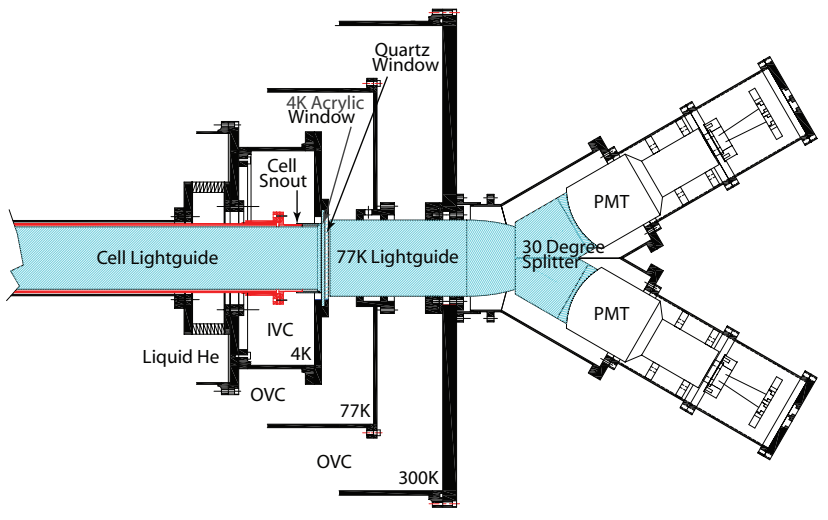

Figure 9: Top view of the light collection system. Note that there are vacuum gaps between the acrylic snout and $4.2 \mathrm{~K}$ window and between the $4.2 \mathrm{~K}$ window and the $77 \mathrm{~K}$ to $300 \mathrm{~K}$ lightguide.

an aluminum flange that makes an indium seal to the cell end cap as well as provides a mating surface for an acrylic window assembly.

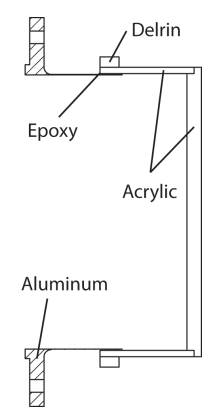

Figure 10: Schematic of the cell window assembly. The aluminum is machined from a single piece of material with a thin cylindrical fin extending towards the acrylic. The acrylic is epoxied to the this fin, providing stress relief due to the differences in thermal contraction.

The assembly is constructed from a $12.8 \mathrm{~cm}$ diameter circular acrylic disk that is first epoxied using Stycast 1266[7] to a $4.2 \mathrm{~cm}$ long, $12.8 \mathrm{~cm}$ outer-diameter, $12.2 \mathrm{~cm}$ inner-diameter acrylic tube. A small lip is machined in the circular acrylic disk to center the window. The acrylic tube is then epoxied around the outside of a thin aluminum fin with a wall thickness of $0.25 \mathrm{~mm}$. The outer diameter of the aluminum tube is machined to be $0.1 \mathrm{~mm}$ smaller than the measured inner diameter of the acrylic tube. The epoxy surfaces are roughened slightly with sandpaper. The total length of the aluminum fin is $3 \mathrm{~cm}$, of which about $1 \mathrm{~cm}$ overlaps with the acrylic. The snout assembly is sealed to the cell endcap using a standard indium seal.

After epoxying the acrylic window to the acrylic tube, the assembly was annealed in an oven at $80{ }^{\circ} \mathrm{C}$ for 4 hours. Afterwards, the temperature was lowered back to room temperature over 8 hours. This treatment relieved the residual stresses due to machining as well as fully formed all cross-links in the epoxy.

The Stycast 1266 epoxy joint between the aluminum flange and acrylic was then made by filling the joint using capillary action. We found it necessary to mix the epoxy an hour or two prior to use in order to reduce the viscosity when used at room temperature. The entire snout assembly was again annealed using the above procedure.

The aluminum to acrylic joint was modeled using Ansys finite element analysis. These simulations showed that when cooled, the joint had the tendency to peel up at the end of the acrylic. To counter this action a ring of Delrin[7] was placed around the outside of the joint as can be seen in Figs. 10 and 11 to improve the contact along the joint. Note that we have also successfully constructed flanges of a smaller size using 70-30 cupronickel in addition to aluminum. The entire assembly remained leak-tight after multiple thermal cycles.

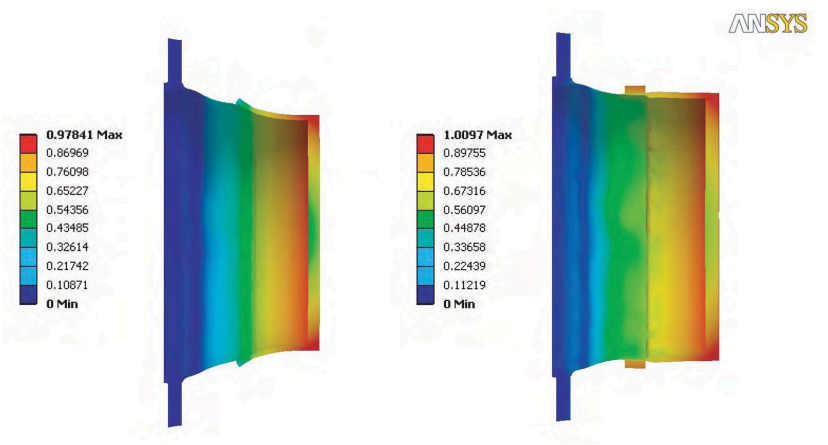

Figure 11: Finite element analysis of the thermal expansion in the cell optical window shows peeling of the acrylic at the aluminum to acrylic epoxy joint (left). This was relieved by adding a Delrin ring at this location (right). The color scale represents the strain in $\%$ of $\Delta L / L$.

The acrylic window at $4.2 \mathrm{~K}$ makes an indium seal to the stainless steel flange separating the outer vacuum can from the inner vacuum can. It also blocks the blackbody radiation. However, acrylic is a poor thermal conductor. Without the quartz window, the center of the acrylic window heats up to $>20 \mathrm{~K}$, causing a large radiative heat load onto the measurement cell. Although the single-crystal quartz window has a lower cutoff wavelength for blackbody radiation, it has much higher thermal conductivity. It is pressed onto the $77 \mathrm{~K}$ facing side of the acrylic window to both partially block blackbody radiation to lower radiative heating as well as cool the acrylic window.

The light then travels through a $14 \mathrm{~cm}$ diameter, $26 \mathrm{~cm}$ long cast UVT-grade acrylic rod that extends from $77 \mathrm{~K}$ to $300 \mathrm{~K}$. Using thermal conductivities of acrylic of $0.19 \mathrm{~W} / \mathrm{mK}$ at room temperature and $0.16 \mathrm{~W} / \mathrm{mK}$ at $77 \mathrm{~K}[22]$, an ANsYs finite element model was used to optimize the boundary conditions created by thermal clamps for the light guide which generate the thermal gradients from one end to the other. Two flanges were precisely machined to have an inner diameter smaller than the outer diameter of the acrylic rod in order to ensure the full thermal contact at $77 \mathrm{~K}$ and a vacuum seal at $300 \mathrm{~K}$. The rod was then cooled to $77 \mathrm{~K}$ such that thermal contraction allowed it to slide through the room temperature flanges. However, upon simultaneous cooling of the aluminum and the acrylic, the dimensions are such that full contact of the mating surfaces will remain until temperatures are well below $77 \mathrm{~K}$. Upon repeated vacuum, and cooldown cycling this guide element has reliably 
performed its functions. A photograph of the assembly is shown in Fig. 12.

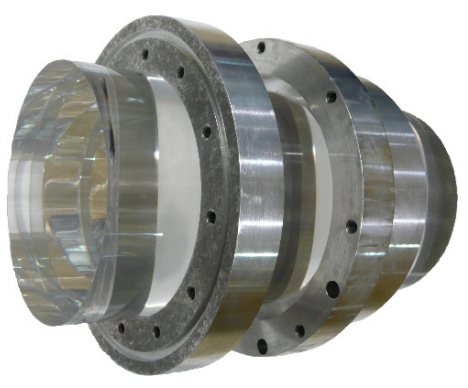

Figure 12: Photograph of the $14 \mathrm{~cm}$ diameter, $26 \mathrm{~cm}$ long $77 \mathrm{~K}$ light guide element. Photon signals enter from the right in this picture, and exit toward the photomultiplier tubes on the left.

In previous designs, the $77 \mathrm{~K}$ thermal connections to the acrylic lightguide that extends from $300 \mathrm{~K}$ to $77 \mathrm{~K}$ was not sufficient to cool the center of the light guide. This resulted in a significant radiative heat load to $4.2 \mathrm{~K}$. In the present design, this was minimized by thermally clamping the lightguide across a longer area of the guide and displaced from the end. The position of this clamp was optimized using Ansys thermal simulations to insure that the center of the acrylic guide approached $77 \mathrm{~K}$ at equilibrium. Less than $0.14 \mathrm{~W}$ of additional radiative heat loads are expected from the guide. The heat load to the cell from the neutron entrance windows is quite small.

\section{Performance}

\subsection{Cryostat}

During the design process, the calculated average heat load incident upon the $4.2 \mathrm{~K}$ vessel was $4.8 \mathrm{~W}$, corresponding to a helium usage rate of $163 \mathrm{l} /$ day. The sources of heat input are summarized in Table 2 and arise from blackbody radiation, conduction through the cryogenic posts, conduction to room temperature in each of the vertical towers, operation of the dilution refrigerator, heat transfer through the current leads, and from eddy current heating in the solenoids during magnetic field ramps. The two cryocoolers each provide $1.5 \mathrm{~W}$ of cooling power at $4.2 \mathrm{~K}$, which if fully utilized, reduces the average heat load to $1.8 \mathrm{~W}$, corresponding to a helium boiloff rate of 62 1/day.

The commissioning of the new apparatus took place in two stages. After assembling the dewar without modifications for the beam entrance and light collections systems, and without installation of the magnet and current leads, an initial cooldown was performed to check for cold leaks and measure the cryogen boiloff. During this cooldown, a helium boiloff rate of 100 l/day was observed. This was consistent with the initial estimate of 94 1/day (Table 2) once losses from transfers are taken into account.

After all modifications to the cryostat were complete and the magnets and cryocoolers installed, the helium boiloff rate was measured to be $55 \mathrm{l}$ /day from the magnet bath alone with both
Table 2: Summary of the calculated heat load onto the $4.2 \mathrm{~K}$ can. Note that the Fermilab leads were measured in a separate dewar and contribute 46 1/day of helium boiloff.

\begin{tabular}{|l|c|c|}
\hline Heat Source & $\begin{array}{c}\text { Heat } \\
\text { Input }(\mathrm{W})\end{array}$ & $\begin{array}{c}\text { Duration } \\
(\%)\end{array}$ \\
\hline Blackbody Radiation & 0.8 & 100 \\
Cryogenic Posts & 0.8 & 100 \\
Fermilab HTS leads & 1.4 & 100 \\
Low current HTS leads & 0.1 & 100 \\
Eddy current in solenoids & 5 & 8 \\
Dilution refrigerator & 0.6 & 100 \\
Vertical towers & 0.6 & 100 \\
Lightguides & 0.14 & 100 \\
\hline
\end{tabular}

cryocoolers operating and the magnet not energized. This relied on flowing liquid nitrogen throughout the HTS leads at a rate of $1.7 \mathrm{Nm}^{3} / \mathrm{s}(60 \mathrm{scfm})$; without this flow, the helium boiloff increased to $68 \mathrm{l} /$ day. An additional a boiloff rate of $25 \mathrm{l} /$ day was observed from the bath surrounding the dilution refrigerator. In addition, the operation of the magnets required increased liquid nitrogen cooling $\left(3.4 \mathrm{Nm}^{3} / \mathrm{s}(120 \mathrm{scfm})\right)$ and the boil-off rate increased by an additional $20 \mathrm{l} /$ day. Therefore the total boiloff rate when the magnets are energized was measured to be 100 l/day.

Based on earlier measurements plus the calculated and measured heat loads, the two cryocoolers appear to be reducing the helium consumption by at least $69 \mathrm{l} /$ day, corresponding to a heat load of $2 \mathrm{~W}$. Thus on average, we are utilizing a minimum of $2 / 3$ of the cooling capacity of the cryocoolers. Factors that may impact the cooling include the operation of one cryocooler in an inverted geometry, thermal resistance in the flexible braids connecting the cryocoolers to the helium bath, and unanticipated heat loads from the modifications.

We attribute the additional heat load that arises from the operation of the magnet to ohmic heating. There are four connections within the apparatus connecting the HTS leads to the high-current quadrupole magnet. These connections are all solder joints. The additional $1.2 \mathrm{~W}$ of heating would corresponds to a total resistance of $0.11 \mu \Omega$ across the joints.

To reduce the helium costs, a pulse-tubed based reliquifaction plant with a quoted $18 \mathrm{l} /$ day liquefaction capacity was also used. In practice, however we obtained about 12 1/day liquefaction rate from this system. Additional resources were not available at the time to further reduce the helium consumption. The helium boiloff also had a side effect of reducing the nitrogen boiloff in both dewar towers to effectively zero.

\subsection{Dilution Refrigerator}

The heat load to the measurement cell comes from blackbody radiation, conduction heat from cell supports and sensor wires, neutron beam heating and eddy-current heating. The estimated heat load from each source is summarized in Table 3.

Eddy current heating has the largest peak heat input. Fortunately, it only happens during magnet ramps (dictated by the experimental measurement process) that take place $8 \%$ of the 
Table 3: Summary of estimated cell heat loads. The eddy current calculation assumes a magnet ramp time of $200 \mathrm{~s}$. Duration is the fraction of time when the heat source is on.

\begin{tabular}{|l|c|c|}
\hline Heat Source & $\begin{array}{c}\text { Heat } \\
\text { Input }(\mu \mathrm{W})\end{array}$ & $\begin{array}{c}\text { Duration } \\
(\%)\end{array}$ \\
\hline Blackbody radiation & 48 & 100 \\
cell supports and sensor wires & 1 & 100 \\
neutron beam & 15 & 40 \\
Eddy current in cell wall $(\mathrm{CuNi})$ & 1000 & 8 \\
Eddy current in buffer cell $(\mathrm{Cu})$ & 1200 & 8 \\
\hline
\end{tabular}

total running time. The average calculated heat load is $182 \mu \mathrm{W}$, with peak heatload of $2.3 \mathrm{~mW}$ during magnet ramps.

The cell is connected to the dilution refrigerator through a $2.54 \mathrm{~cm}$ diameter superfluid heat link as discussed in section 4.3. Using the calculated conductivity of $0.8 \mathrm{~W} / \mathrm{K} \cdot \mathrm{cm}$ and the measured cooling power of the dilution refrigerator, we calculate that the temperature of the bulk liquid helium inside the cell should be about $100 \mathrm{mK}$. The actual measured temperatures during data collection were closer to $250 \mathrm{mK}$. This difference is likely a result of larger radiative heat loads through either the neutron entrance windows or light collection system. Because of the large diameter of the cell and high thermal conductivity of the helium, there should virtually be no temperature gradient across the cell. When ramping the magnetic field, about $0.4 \mathrm{~J}$ of energy is deposited into the cell through eddy-current heating, which raises the cell temperature briefly to a little over $300 \mathrm{mK}$, but cools back to the base temperature of $250 \mathrm{mK}$ in several hundred seconds.

Beam heating arises primarily from neutron capture in the boron shielding material. Alpha particles from the ${ }^{10} \mathrm{~B}(n, \alpha)$ reaction $(Q=2.79 \mathrm{MeV})$ deposit all of their kinetic energy into the cell. Neutrons scattered and absorbed in the acrylic light guide however produce gamma rays via the reaction $p(n, \gamma) d$, where the majority of the gamma rays leave the measurement cell. About $1 \%$ of the $0.89 \mathrm{~nm}$ neutrons will scatter in the helium, with the remainder incident upon the acrylic light guide. A small fraction of the neutrons incident upon the acrylic will backscatter and re-enter the trapping region. We observe a temperature rise of $10 \mathrm{mK}$ during beam-on operation, corresponding to an equivalent heat load of $15 \mu \mathrm{W}$. Therefore we estimate that roughly $10 \%$ of the neutrons are absorbed in the BN shielding. Note that if all neutrons were absorbed in the BN, the heat load would be $175 \mu \mathrm{W}$.

Similar to the $4.2 \mathrm{~K}$ heatload above, the heat load to the cell from the neutron entrance windows is also quite small due to the thermal conductivity of the Be window at 4.2 K. Similarly, the combination of the quartz and acrylic windows at $4.2 \mathrm{~K}$ minimize the radiative heat load from the light collection end. Note that when the light guides are not connected to the PMTs and room light enters, we observe about a $20 \mathrm{mK}$ rise in the cell temperature.

During liquefaction of helium in the cell at temperatures between $4.2 \mathrm{~K}$ and above $800 \mathrm{mK}$, it was observed that good thermal contact is maintained between the cell and the mix- ing chamber. This good thermal conductivity is attributed to convection in the helium gas above the liquid due to the relatively high saturated vapor pressures at these temperatures. Below $800 \mathrm{mK}$, it is necessary to have the helium level within the buffer cell to maintain thermal contact between the cell and the mixing chamber.

\section{Conclusions}

The magnetic trapping apparatus was successfully operated for continuous periods as long as six months at the $0.89 \mathrm{~nm}$ monochromatic neutron beamline at NIST. Using this apparatus, we were able to conclusively demonstrate the magnetic trapping of ultracold neutrons and are in the process of using this apparatus to measure the beta-decay lifetime of the neutron. Works to significantly reduce both statistical and systematic uncertainties are underway.

\section{Acknowledgments}

We thank G. Seidel, D. Haase, and R. Golub for useful cryogenic discussions. We acknowledge the support of the NIST, US Department of Commerce, in providing support, including the neutron facilities used in this work. This work is also supported in part by the US National Science Foundation under Grant No. PHY-0855593 and the US Department of Energy under Grant No. DE-FG02-97ER41042.

\section{References}

[1] S. Burles et al., Phys. Rev. Lett. 82, 4176 (1999).

[2] R. E. Lopezand M. S. Turner, Phys. Rev. D 59, 103502 (1999).

[3] N. Severijns, M. Beck, and O. Naviliat-Cuncic, Rev. Mod. Phys. 78, 991 (2006).

[4] F. E. Wietfeldt and G. L. Greene, Rev. Mod. Phys. 83, 1173 (2011).

[5] P. R. Huffman et al., Nature 403, 62 (2000).

[6] C. R. Brome et al., Phys. Rev. C 63, 055502 (2001).

[7] Any mention of commercial products or reference to commercial organizations is for information only; it does not imply recommendation or endorsement by NIST nor does it imply that the products mentioned are necessarily the best available for the purpose.

[8] G. Citver, Proc. of the 1999 Particle Accelerator Conf., 1420 (1999).

[9] L. Yang et al., Rev. Sci. Instrum. 79, 031301 (2008).

[10] K. Tsuchiya, K. Egawa, K. Endo, Y. Morita, and N. Ohuchi, IEEE Trans. Magn. 27, 1940 (1991).

[11] J. M. Lock, Cryogenics 9, 438 (1969).

[12] K. Sasaki et al., IEEE Trans. Appl. Superconductivity, 2570 (1997).

[13] A. T. Visser, EPE '91, $4^{\text {th }}$ European Conference on Power Electronics and Applications, P. Ferraris, editor, 521 (1991).

[14] T. Nicol et al., Adv. in Cryo. Eng. 33, 227 (1988).

[15] F. Pobell, Matter and Methods at Low Temperatures, 2nd edition, Springer-Verlag, Berlin, 1996.

[16] D. S. Greywall, Phys. Rev. B 23, 2152 (1981).

[17] P. R. Huffman et al., J. Lumniescence 92, 291 (2000).

[18] S. N. Dzhosyuk et al., Nucl. Instrum. Meth. B 217, 457 (2004).

[19] H. Fujishiro et al., Jap. J. of App. Phys. 36, 5633 (1997).

[20] P. Wikus et al., Cryogenics 48, 515 (2008).

[21] J. S. Butterworth et al., Rev. Sci. Inst. 69, 3998 (1998).

[22] J. Ekin, Experimental Techniques for Low-Temperature Measurements, Oxford University Press, (2006) 